\title{
Bacteria Producing Extended Spectrum $\beta$-lactamases (ESBLs) in Hospitalized Patients: Prevalence, Antimicrobial Resistance Pattern and its Main Determinants
}

\author{
Mehdi Yousefipour ${ }^{1}$, Mehrnaz Rasoulinejad ${ }^{2}$, Azar Hadadi $^{3}$, Negin Esmailpour ${ }^{4}$, Alireza Abdollahi ${ }^{* *}$, \\ Sirous Jafari ${ }^{6}$, Atieh Khorsand ${ }^{7}$ \\ 1. Dept. of Infectious and Tropical Diseases, Imam Khomeini Hospital Complex, Tehran University of Medical Sciences, \\ Tehran, Iran \\ 2. Professor, Dept. of Infectious Diseases, Imam Khomeini Complex Hospital, Tehran University of Medical Sciences, \\ Tehran, Iran \\ 3. Professor, Sina Hospital, Tehran University of Medical Sciences, Tehran, Iran \\ 4. Associate Professor, Dept. of Infectious Diseases, Shariati Hospital, Tehran University of Medical Sciences, Tehran, \\ Iran \\ 5. Professor, Dept. of Pathology, Imam Khomeini Hospital Complex, School of Medicine, Tehran University of Medical \\ Sciences, Tehran, Iran \\ 6. Associate Professor, Dept. of Infectious Diseases, Imam Khomeini Complex Hospital, Tehran University of Medical \\ Sciences, Tehran, Iran \\ 7. Dept. of Pathology, Shariati Hospital Complex, School of Medicine, Tehran University of Medical \\ Sciences, Tehran, Iran
}

\begin{tabular}{c}
\hline KEYWORDS \\
\hline beta-Lactamase, \\
Antibiotic Resistance \\
Hospital-Patient \\
\hline Article Info
\end{tabular}

Received 30 March 2018; Accepted 24 Dec 2018; Published Online 27 Dec 2018;

Doi: 10.30699/IJP.14.1.61

\section{ABSTRACT}

Background and Objective: There is a growing concern regarding the lack of new antibiotics, especially for multidrug-resistant bacteria that produce Extended Spectrum $\beta$-Lactamases (ESBLs). The present study aims to assess the prevalence of bacteria producing ESBLs, their antimicrobial resistance pattern, and its main determinants among hospitalized patients.

Methods: The study population included 383 consecutive patients with a definitive diagnosis of urinary tract infection (UTI). All eligible subjects for the study had a positive culture for gram-negative microorganisms in urine specimens. ESBL producing isolates were characterized phenotypically for ESBL production using the double disc synergy test.

Results: In total, 383 specimens were assessed, among which 212 (55.4\%) were related to bacteria producing ESBLs (ESBL+). Of those with ESBL + infections, $65.5 \%$ were sourced from catheters (as hospital-associated UTIs), and 35.5\% were categorized as community-associated UTIs. In the group consisting of bacteria producing ESBLs, the highest sensitivity was observed with Imipenem (72.2\%), while the highest resistance was revealed with ceftriaxone (100\%).

Conclusion: We have shown that our community faces a high prevalence of bacteria producing ESBLs, mostly sourced from the catheterization of hospitalized patients. The highest bacterial sensitivity was observed with Imipenem, nitrofurantoin, and amikacin, while the highest resistance was found with ceftriaxone and cotrimoxazole, suggesting the ineffectiveness of using the two latter antibiotics for eradicating these bacterial infections. On the other hand, a history of urinary catheterization and previous hospitalization were two main determinants of their presence, a finding which emphasizes the importance of avoiding catheterization and hospitalization of patients with UTIs without proper indications.
Corresponding Information:

Alireza Abdollahi, Professor, Department of Pathology, Imam Khomeini Hospital Complex, School of

Medicine, Tehran University of Medical sciences, Tehran, Iran.

Email: dr_p_abdollahi@yahoo.com

Copyright (c) 2019. This is an open-access article distributed under the terms of the Creative Commons Attribution-noncommercial 4.0 International License which permits copy and redistribute the material just in noncommercial usages, provided the original work is properly cited. 


\section{Introduction}

The bacteria that infect the critically ill patients admitted into hospitals, especially into intensive care units, widely vary in terms of antibiotic resistance and genotypic pattern. Bacterial drug resistance is now a major concern leading to high morbidity, and even mortality, especially in third world countries (1). Due to the time-consuming nature of the laboratory diagnoses of these microbial agents, delays in diagnosis may lead to delays in proper antimicrobial therapy. There is also a growing concern regarding the lack of new antibiotics, especially for multidrug-resistant bacteria that produce ESBLs $(2,3)$. The beta-lactamases produced by the Enterobacteriaceae family of gram-negative organisms, in particular Klebsiella pneumonia and Escherichia coli are hydrolytic enzymes that confer bacterial resistance to $\beta$-lactam antibiotics, such as the penicillin and cephalosporin families that are common antimicrobial drugs found all around the world $(4,5)$. The bacteria producing ESBLs have become a major cause of nosocomial infection, particularly in the ICU, with the majority of ESBL producers being isolated from critical care patients (6). Some risk factors have been identified that render patients prone to community-associated ESBL infections, including old age, being female, diabetes mellitus, previous antibiotic usage, recurrent urinary tract infections, and prior instrumentation to urinary tract $(7,8)$. Although a downward trend of infections due to ESBL-producing bacteria has been recently reported in developed countries, this trend has remained constant, or even increased in some developing countries (9). Unfortunately, the incidence of bacteria producing ESBLs remains uncertain. Hence, the present study aimed to assess the prevalence of bacteria producing ESBLs, their antimicrobial resistance pattern, and its main determinants among hospitalized patients in a great referral hospital in Iran between 2016 and 2017.

\section{Materials and Methods}

The study targeted three endpoints included to determine the overall prevalence of ESBL $(+)$ bacte- ria, to assess the antimicrobial resistance patterns of these species, and to determine the main predictors of the presence of these bacteria. In this regard, a biphasic study was planned as a cross-sectional study to achieve two former endpoints and a case-control study with the aim of comparing baseline variables between the two groups, with and without bacteria producing ESBLs. The study population included 383 consecutive patients, with a definitive diagnosis of urinary tract infections (UTIs), who were referred to Imam Khomeini hospital in Tehran within a period of five months. All eligible subjects for the study had a positive culture for gram negative microorganisms in urine specimens. Hospitalized patients over 18 years of age were categorized into two groups according to the criteria of the CDC guidelines as having 1) nosocomial UTIs: infections related to catheter insertion within hospitalization that was defined as a) having urine catheters for more than 2 days; b) having at least one of the following symptoms: fever (higher than $38^{\circ} \mathrm{C}$ ), suprapubic tenderness, tenderness in flank or abdomen, urinary urgency, frequency, or emergency; c) urine culture with a colony count higher than 105 colonies. 2) community-acquired UTIs: hospitalization due to UTIs with manifesting symptoms upon admission: including fever, urinary frequency, dysuria, urinary urgency, flank or suprapubic tenderness, and laboratory findings of UTIs including urine culture a colony count exceeding 105 colonies. All urinary specimens were collected mid-stream using the clean catch method via Foley catheter and were cultured in Mueller Hinton agar medium at $35^{\circ} \mathrm{C}$. Standard methods for isolation and identification of these bacteria were used. ESBL-producing isolates were characterized phenotypically for ESBL production by using the Double Disc Synergy Test (DDST) as recommended by the Clinical Laboratory Standards Institute (CLSI) (10). The test was done by using cefotaxime $(30 \mu \mathrm{g})$ and ceftazidime $(30 \mu \mathrm{g})$, both alone and in combination with clavulanic acid. A $>5 \mathrm{~mm}$ increase in zone diameter for either antimicrobial agents tested in combination with clavulanic acid, versus its zone when tested alone, was taken 
as positive result for ESBL production. To assess the factors that might be associated with the susceptibility to the infection by ESBL + bacteria, the baseline characteristics and clinical information of the patients, including demographics, medical history, medications, and laboratory parameters, were collected by reviewing hospital records.

Results were presented as a mean \pm standard deviation (SD) for quantitative variables and were summarized by absolute frequencies and percentages for categorical variables. The normality of data was analyzed using the Kolmogorov-Smirnoff test. Categorical variables were compared using the chisquared test or Fisher's exact test when more than $20 \%$ of cells with an expected count of less than 5 were observed. Quantitative variables were also compared with the t-test or the Mann-Whitney U test. The main determinants of the ESBL $(+)$ condition were assessed using multivariable logistic regression modeling. For the statistical analysis, the statistical software SPSS version 16.0 for windows (SPSS Inc., Chicago, IL) was used. P values of 0.05 or less were considered statistically significant.

\section{Results}

We assessed 383 specimens with definitive diagnoses of UTI, out of which $212(55.4 \%)$ cases were $\operatorname{ESBL}(+)$.

The male to female ratio in the ESBL $(+)$ group, compared to the ESBL (-) group, was significantly higher $(P=0.002)$ (Table 1$)$. There was no difference in mean age between these two groups $(50.80$ \pm 22.24 years versus $47.79 \pm 25.64$ years, $P=0.220$ ) (table 1).

With regards to past medical history, there were more patients with diabetes in the $\operatorname{ESBL}(+)$ group compared to the ESBL(-) group (10.9\% versus $2.9 \%$, $P=0.003$ ), the same as their history of cancer and chemotherapy $(20.8 \%$ versus $11.1 \%, P=0.011)$, renal failure and dialysis $(14.2 \%$ versus $5.8 \%$, $P=0.041)$, ICU admission ( $22.1 \%$ versus $9.3 \%, P=$ 0.001 ), long hospital admission of over seven days $(14.1 \%$ versus $5.9 \%, P=0.008)$ and antibiotic con- sumption in the past 3 months (16\% versus $2.9 \%, P$ $<0.001)$. A history of UTI in the past three months was also more in the group with bacteria producing ESBLs $(P=0.012)$.

However, there was no between-group difference in the history of ischemic heart diseases, CVA and previous surgeries in the urinary tract in the past 1 year.

The Foley catheter was significantly more used in the ESBL $(+)$ group $(61.8 \%$ versus $23.4 \%, P=$ $0.001)$. On the other hand, $65.5 \%$ of cases of the ESBL $(+)$ group were sourced from catheters (as hospital-associated UTIs), and 35.5\% were categorized as community-associated UTIs.

No difference was found in the prevalence of some species, such as E. coli, between two groups (64.6\% versus $66.1 \%$ ), while some other species. such as Klebsiella, were more prevalent in the ESBL $(+)$ group (30.2\% versus $21.6 \%)$.

In the group consisting of bacteria producing ESBLs, the highest sensitivity was observed with Imipenem (72.2\%), followed by nitrofurantoin $(69.3 \%)$, amikacin (69.3\%), and gentamycin (38.7\%) (Figure 1), while the highest resistance was revealed to be with ceftriaxone (100\%) and cotrimoxazole $(91.0 \%)$. As shown in Figure 1, the resistance to all types of antibiotics was considerably higher in the ESBL (+) group compared with the other group. Using the multivariate logistic regression model, and with the presence of potential confounders, the history of using Foley catheter $(\mathrm{OR}=3.761, P<0.001)$ and history of hospitalization $(\mathrm{OR}=5.590, P<0.001)$ were the main predictors of the presence of ESBLproducing bacteria. 
Table 1. Baseline characteristics between the groups with and without bacteria producing ESBLs

\begin{tabular}{|c|c|c|c|}
\hline Item & ESBL (+) group & ESBL (-) group & $P$-value \\
\hline Male & $40.1 \%$ & $25.1 \%$ & 0.002 \\
\hline Mean age, year & $50.80 \pm 22.24$ & $47.79 \pm 25.64$ & 0.220 \\
\hline \multicolumn{4}{|l|}{ Bacterial species } \\
\hline E. Coli & $64.6 \%$ & $66.1 \%$ & 0.879 \\
\hline Kelebsiella & $30.2 \%$ & $21.6 \%$ & 0.001 \\
\hline Citrobacter & $4.2 \%$ & $2.9 \%$ & 0.166 \\
\hline Enterobacter & $0.0 \%$ & $5.3 \%$ & 0.098 \\
\hline Morganella & $0.0 \%$ & $0.6 \%$ & 0.759 \\
\hline Proteus & $0.0 \%$ & $2.9 \%$ & 0.456 \\
\hline Other enterobacteriace & $0.9 \%$ & $0.6 \%$ & 0.579 \\
\hline Foley catheter used & $61.8 \%$ & $23.4 \%$ & 0.008 \\
\hline Renal failure and dialysis & $14.2 \%$ & $0.6 \%$ & 0.041 \\
\hline Ischemic heart disease & $4.2 \%$ & $2.3 \%$ & 0.307 \\
\hline Cerebrovascular disease & $3.3 \%$ & $2.3 \%$ & 0.576 \\
\hline cancers + chemotherapy & $20.8 \%$ & $11.1 \%$ & 0.011 \\
\hline Diabetes mellitus & $10.9 \%$ & $2.9 \%$ & 0.003 \\
\hline $\begin{array}{c}\text { Surgery of urinary tract in past one } \\
\text { Year ago }\end{array}$ & $3.8 \%$ & $2.3 \%$ & 0.424 \\
\hline Admission more than 7 days & $14.1 \%$ & $5.9 \%$ & 0.008 \\
\hline History of hospitalization in past $3 \mathrm{mo}$ & $47.2 \%$ & $11.1 \%$ & $<0.001$ \\
\hline History of antibiotic consumption in past $3 \mathrm{mo}$ & $16 \%$ & $2.9 \%$ & $<0.001$ \\
\hline History of UTI in past 3 mo & $13.7 \%$ & $5.9 \%$ & 0.012 \\
\hline History of ICU admission & $22.1 \%$ & $9.3 \%$ & 0.001 \\
\hline
\end{tabular}




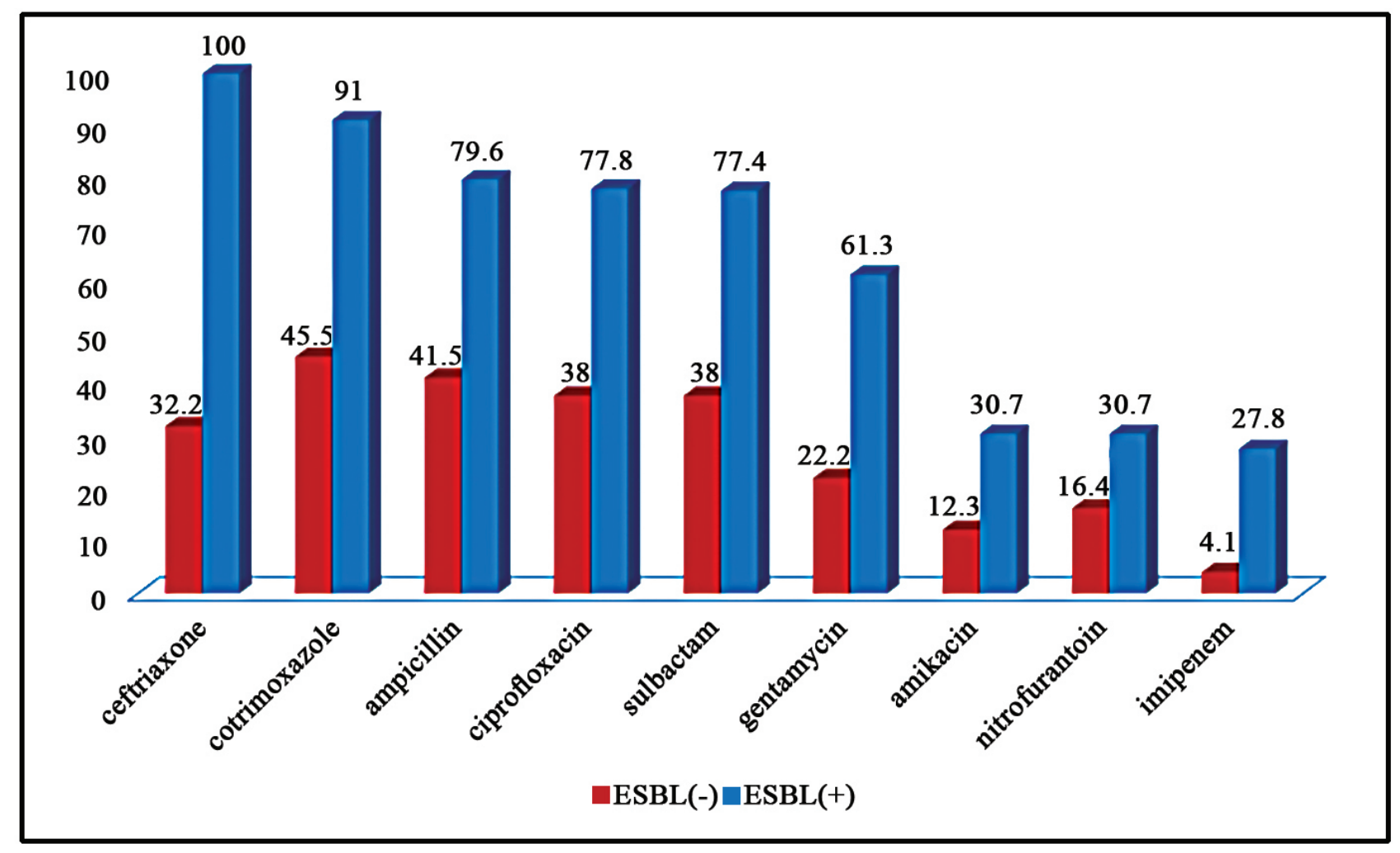

Figure1. The resistance with different antibiotics in ESBLs (+) and ESBL (-) groups

\section{Discussion}

The proliferation of ESBL-producing bacteria is an important alarm for all countries around the world, due to their high resistance to various antimicrobial agents. In this regard, different factors such as underlying chronic disorders, a history of hospitalization, malignancies, urinary tract disorders, or urinary catheterization, as well as some mutations in bacterial DNAs, may increase the prevalence of these bacteria, not to mention their resistance to different antibiotics. Thus, the use of proper antibiotics, along with avoiding the overuse or misuse of antibiotics with a high resistance pattern, should be considered to minimize the development of these infections.

In the present study, the overall prevalence of ESBL-producing bacteria was shown to be $55.4 \%$ in our patient population, two-thirds of which was related to urinary catheterization in hospitalized patients, while the rest were community-acquired infections. Secondly, the highest prevalence rate ESBL-producing bacteria was related to E.coli at $64.6 \%$, followed by Klebsiella at $30.2 \%$, which seems to be inconsistent with some previous reports. In a study by Sharma et. al. (11) in 2013 on Indian patients, 52.49\% were found to be ESBL-producing. Also, among various isolates, the highest ESBL production was observed in Klebsiella pneumoniae (67.04\%), followed by Escherichia coli $(56.92 \%)$. In another study conducted in Tanzania, the overall prevalence of ESBLs in all Gram-negative bacteria was $29 \%$. The ESBL prevalence was $64 \%$ in the Klebsiella pneumoniae species and $24 \%$ in E. coli (12).

Frightening figures were also obtained in a study in Mali, where $63 \%$ of adults and $100 \%$ of children were found to carry ESBL-producing Enterobacteriaceae (13). Moreover, in Madagascar, Herindrainy et al. (14) in 2011 observed that $10 \%$ of non-hospitalized patients carried ESBLs. Fatemeh et al. (15) also found that $26.5 \%$ of E. coli and $43 \%$ of Klebsiella pneumoniae were ESBL-positive in their study, which was conducted at the Imam Reza Hospital of Mashhad.

It was also shown that in the group consisting of ESBL-producing bacteria, the highest sensitivity was observed with Imipenem (72.2\%), followed by nitro- 
furantoin $(69.3 \%)$, amikacin (69.3\%) and gentamycin (38.7\%), while the highest resistance was observed with cotrimoxazole (91.0\%) and ceftriaxone (100\%).

In other words, sensitivity with almost all antibiotics was considerably low, even with beta-lactams and macrolides. In this regard, the resistance to cephalosporin and co-trimoxazole was considerably high, revealing the ineffectiveness of these two types of antibiotics in eliminating ESBL-producing bacteria from our hospitalized patients.

In a Sharma et al. survey (15), the sensitivity with Imipenem was found to be at $100 \%$, and the highest sensitivity was shown to be with beta-lactams and aminoglycosides. Another investigation was conducted at a tertiary hospital in Nigeria by Ruth et al (16), in which the ESBL isolates showed high resistance to tetracycline, gentamicin, pefloxacin, ceftriaxone, cefuroxime, ciprofloxacin and Augmentin. In a study by Majda et al (17), maximum resistance was recorded in E. coli as cefotaxime (98.9\%), Ceftazidime (96.7\%) and Cefuroxime (93.4\%) while minimum resistance was seen with Imipenem (0.8\%), fosfomycin (1.2\%) and nitrofurantoin as well piperacillin/tazobactam (2.2\%) each.

The ESBL-producing Klebsiella showed maximum resistance to ceftazidime (100\%), cefotaxime (89\%), and Cefuroxime (84\%) while minimum resistance was seen with Imipenem (4\%), Nitrofurantoin and Piperacillin/Tazobactam (8\%).

In another study, Shakti et al (18) showed a progressive increase in drug resistance against each antibiotic, with the maximum resistance values recorded against gentamycin: $92 \%$ and $79 \%$, oxacillin: $94 \%$ and $69 \%$, ceftriaxone: $85 \%$ and $58 \%$, and Norfloxacin $97 \%$, and $69 \%$, both in nosocomial and com- munity isolates respectively.

Our study identified these conditions as risk factors for developing $\operatorname{ESBL}(+)$ infections: Foley catheterization, renal failure and dialysis, cancer and chemotherapy, ICU admission, DM, long admission exceeding 7 days, a history of UTI in the past 3 months, hospitalization in the past 3 months, and a history of antibiotic consumption.

In total, our community faces the high prevalence of ESBL-producing bacteria, most of which originate from catheterization among hospitalized patients. In addition, the highest bacterial sensitivity was related to Imipenem, nitrofurantoin, and amikacin, while the highest resistance was found to be with ceftriaxone and cotrimoxazole. In the end, according to the potential effect of Foley catheterization, and prolonged or repeated hospitalization, on the prevalence rate of ESBL-producing bacterias, it is strongly encouraged to pay attention to the catheterization and hospitalization of patients with UTIs to minimize the number of these bacteria in the community.

\section{Acknowledgements}

The authors would like to thank the staff of the Laboratory Department at the Imam Khomeini Hospital Complex for their devoted technical support, and the Vice Chancellor for Research Affairs at the Tehran University of Medical Sciences. The study was approved by the Ethics Committee of the Tehran University of Medical Sciences (TUMS) (Number: 9211163010).

\section{Conflict of Interest}

The authors declare that there is no conflict of interest in the publication of this paper..

\section{References}

1. Spellberg B, Gilbert DN. The future of antibiotics and resistance: a tribute to a career of leadership by John Bartlett. Clinical infectious diseases. 2014;59(supp1_2):S71-5.

2. Read AF, Woods RJ. Antibiotic resistance management. Evol Med Public Health. 2014;
2014(1):147. https://doi.org/10.1093/emph/eou024 PMID:25355275 PMCID:PMC4228198

3. Lushniak BD. Antibiotic resistance: a public health crisis. Public Health Rep 2014;129(4):314-316. PMID:24982528 PMCID:PMC4037453

4. Queener SF. History and Origins of Beta-Lactam Antibiotics. In: Queener SF, Webber JA, Queener SW, 
67.Bacteria Producing Extended Spectrum $\beta$-lactamases ...

editors. Beta-Lactam Antibiotics for Clinical Use. New York: Marcel Dekker, Inc; 1986.

5. Kong KF, Schneper L, Mathee K. Beta-lactam antibiotics: from antibiosis to resistance and bacteriology. APMIS. 2010;118(1):1-36. https://doi.org/10.1111/ j.1600-0463.2009.02563.x PMID:20041868

6. Shah AA, Hasan F, Ahmed S, Hameed A. Extended-spectrum beta-lactamases (ESbLs): characterization, epidemiology and detection. Crit Rev Microbiol. 2004;30(1):25-32. https://doi. org/10.1080/10408410490266429 PMID: 15116761

7. Rupp ME, Fey PD. Extended spectrum beta-lactamase (ESBL)-producing Enterobacteriaceae: considerations for diagnosis, prevention and drug treatment. Drugs. 2003; 63(4):353-65. PMID: 12558458

8. Susić E. Mechanisms of resistance in Enterobacteriaceae towards beta-lactamase antibiotics. Acta Med Croatica. 2004; 58(4):307-12. PMID: 15700687

9. Pfaller MA, Segreti J. Overview of the epidemiological profileand laboratory detection of extended-spectrum $\beta$-Lactamases. Clinical Infectious Diseases. 2006;42(Supplement_4):S153-63.

10. Clinical Laboratory Standards Institute. Performance standards for antimicrobial disc susceptibility tests, IXX international Supplement (M100- S19). Wayne Pa, USA: National Committee for Clinical Laboratory Standards. 2009.

11. Sharma M, Pathak S, Srivastava P. Prevalence and antibiogram of Extended Spectrum beta-Lactamase (ESBL) producing Gram negative bacilli and further molecular characterization of ESBL producing Escherichia coli and Klebsiella spp. J Clin Diagn Res. 2013;7(10):2173-7. PMID:24298468 PMCID:PMC3843424

12. Moremi N, Mushi MF, Fidelis M, Chalya P, Miram- bo M, Mshana SE. Predominance of multi-resistant gram-negative bacteria colonizing chronic lower limb ulcers (CLLUs) at Bugando Medical Center. BMC Res Notes. 2014;7:211. PMID:24708843 PMCID:PMC3986440

13. Tande D, Jallot N, Bougoudogo F, Montagnon T, Gouriou S, Sizun J. Extended-spectrum beta-lactamase-producing Enterobacteriaceae in a Malian orphanage. Emerg Infect Dis. 2009;15(3):472-4. PMID:19239768 PMCID:PMC2681105

14. Herindrainy $\mathrm{P}$, Randrianirina F, Ratovoson R, Ratsima Hariniana E, Buisson Y, Genel N, et al. Rectal carriage of extended-spectrum beta-lactamaseproducing gram-negative bacilli in community settings in Madagascar. PLoS One. 2011;6(7):e22738. PMID:21829498 PMCid:PMC3146483

15. Fatemeh A, Emran A, Elnaz K, Mohammad J G S, Mahboubeh N. The frequency of extended spectrum beta lactamase (ESBL) in Escherichia coli and Klebsiella pneumonia: a report from Mashhad, Iran. J Med Bacteriol. 2012;1(3):12-19.

16. Ruth A.A, Damian C.O, Romanus I.I, Charles O.E. Antimicrobial resistance status and prevalence rates of extended spectrum beta-lactamase producers isolated from a mixed human population. Bosnian J Basic Med Sci. 2011;11(2):91-96.

17. Majda Q, Najma A, Summyia B. Evaluation of extended spectrum beta-lactamase mediated resistance in Escherichia coli and Klebsiella in urinary tract infection at a tertiary care hospital. Biomedica .2013;29:78-81.

18. Shakti R, Debasmita D, Mahesh C, Sahu R, Padhy N. Surveillance of ESBL producing multidrug resistant Escherichia coli in a teaching hospital in India. Asian Pac J Trop Dis. 2014;4(2):140-149. https:// doi.org/10.1016/S2222-1808(14)60331-5

\section{How to Cite This Article}

Yousefipour M, Rasoulinejad M, Hadadi A, Esmaeilpour N, Abdollahi A, Jafari S, khorsand A. Bacteria Producing Extended Spectrum $\beta$-lactamases (ESBLs) in Hospitalized Patients: Prevalence, Antimicrobial Resistance Pattern and its Main Determinants. Iranian Journal of Pathology. 2019; 14(1): 61-67. 